Scientific

Papers 


\title{
Observations of candidate oscillating eclipsing binaries and two newly discovered pulsating variables
}

\author{
A. Liakos, and P. Niarchos \\ National and Kapodistrian University of Athens, Faculty of Physics, \\ Department of Astrophysics, Astronomy and Mechanics, \\ GR 157 84, Zografos, Athens, Greece
}

\begin{abstract}
CCD observations of 24 eclipsing binary systems with spectral types ranging between $\mathrm{A} 0-\mathrm{F} 0$, candidate for containing pulsating components, were obtained. Appropriate exposure times in one or more photometric filters were used so that short-periodic pulsations could be detected. Their light curves were analyzed using the Period04 software in order to search for pulsational behaviour. Two new variable stars, namely GSC 2673-1583 and GSC 3641-0359, were discovered as by-product during the observations of eclipsing variables. The Fourier analysis of the observations of each star, the dominant pulsation frequencies and the derived frequency spectra are also presented.
\end{abstract}

Accepted: 2009, March 16

Individual Objects: AD And, RY Aqr, UW Boo, AL Cam, AY Cam, TY Cap, EG Cep, RW Cet, WY Cet, BR Cyg, KR Cyg, V456 Cyg, V466 Cyg, V1034 Cyg, RZ Dra, Z Dra, TZ Eri, GSC 2673-1583, GSC 3641-0359, TX Her, V338 Her, V359 Her, IQ Per, GH Peg, X Tri, RS Tri

\section{Introduction and Observations}

The subject of the present study is a search for $\delta$ Scuti components in several detached and semi-detached systems. Most of these components were selected from the list of Soydugan et al. (2006). Mkrtichian et al. (2004) introduced the oEA (oscillating EA) stars as the (B)A-F spectral type mass-accreting mainsequence pulsating stars in semi-detached Algol-type eclipsing binary systems. 
In general, in order to search photometrically for possible short periodic pulsations in a single star or a binary system, the following observational guidelines should be followed: i) the span time should be longer than $2.5 \mathrm{hrs}$, since the majority of the pulsation periods of $\delta$ Scuti stars range between 20 min- 8 hrs (Soydugan et al. 2006), ii) the B-filter should be used since the pulsations have their highest amplitude in this spectral range, given that the pulsating components are of early spectral type (A2-F2). For the brighter systems, other filters could also be used. iii) the comparison star should be bright enough in order to decrease the standard deviation of each observation, iv) appropriate exposure times must be used in order to achieve a photometric SNR (signal-to-noise ratio) higher than 100 .

In addition to the general observational guidelines mentioned above, the relatively small diameter $(0.4 \mathrm{~m})$ of the telescope used for the observations of the present work, puts an upper limit of 11 mag for the observations in the B-filter.

The observations were carried out during June 2007-December 2008 at the Gerostathopoulion Observatory of the University of Athens, using the $40 \mathrm{~cm}$ Cassegrain telescope equipped with the ST-8XMEI CCD camera and the BVRI Bessell photometric filters.

In some cases (AD And, AL Cam, X Tri), although the results for possible pulsational behaviour were negative (see table 1 ), the observations were continued in order to obtain complete light curves which will be used for future work, unrelated to the present study.

The star GSC 2673-1583 $\left(\alpha_{2000}=19^{h} 54^{m} 39.5^{s}, \delta_{2000}=+32^{\circ} 56^{\prime} 2.7^{\prime \prime}\right.$, $\left.V_{\text {mag }}=10.8\right)$ was detected as a variable by Liakos \& Niarchos (2008) in the field of view of V466 Cyg and it was observed during August and September of 2008 for 6 nights using the B-filter. The star GSC 3641-0359 $\left(\alpha_{2000}=\right.$ $23^{h} 35^{m} 50^{s}, \delta_{2000}=+48^{\circ} 43^{\prime} 43^{\prime \prime}, V_{\text {mag }}=11.3$ ) was observed using BVRI filters for 5 nights during the same time period, while its variability was also discovered by the authors in the field of view of AD And. The finding charts of both stars are presented in figure 2 .

\section{Results of frequency analyses}

Differential magnitudes were obtained for all systems using the software Muniwin v.1.1.23 (Hroch 1998). We performed frequency-analysis of all the available observational points in the interval from 5 to $80 \mathrm{c} / \mathrm{d}$ (typical range for $\delta$ Scuti stars, Breger 2000) with the software PERIOD04 which is based on the classical Fourier analysis (Lenz \& Breger 2005). In table 1 we list the name of the system given in the GCVS catalog, the apparent magnitude and the spectral type of the system, the filters used, the number of nights of observations, the total 
Table 1: The log of observations and the results for the possible pulsational behaviour of the selected 24 eclipsing binaries

\begin{tabular}{|c|c|c|c|c|c|c|c|c|c|}
\hline System & $\begin{array}{c}\text { appar. } \\
\text { mag }\end{array}$ & $\begin{array}{l}\text { Spec. } \\
\text { type }\end{array}$ & filters & nights & $\begin{array}{c}\text { time span } \\
\text { (hrs) }\end{array}$ & $\begin{array}{c}\text { SD } \\
(\mathrm{mmag})\end{array}$ & $\begin{array}{c}\text { phase } \\
\text { interval }\end{array}$ & $f_{\text {dom }}$ & ref. \\
\hline AD And & $11.2(\mathrm{~V})$ & $\mathrm{A} 0$ & BVRI & 5 & 24 & 4.3 & 0.01 .0 & - & 2 \\
\hline RY Aqr & $8.9(\mathrm{~V})$ & A8 & B & 1 & 3 & 5.4 & $0.18-0.25$ & - & 1 \\
\hline UW Boo & $10.4(\mathrm{~B})$ & F0 & B & 1 & 5 & 5.7 & $0.43-0.63$ & - & 1 \\
\hline AL Cam & $10.3(\mathrm{~V})$ & A2 & BV & 7 & 34 & 6.8 & $0.0-1.0$ & - & 1 \\
\hline AY Cam & 9.9 (B) & - & BV & 1 & 6 & 4.9 & $0.73-0.82$ & - & - \\
\hline TY Cap & $10.3(\mathrm{~V})$ & A5 & BVRI & 12 & 37 & 5.2 & $0.0-1.0$ & $24.2 \mathrm{c} / \mathrm{d}$ & 1 \\
\hline EG Cep & $9.5(\mathrm{~V})$ & A3 & BVR & 2 & 13 & 4.1 & $0.0-1.0$ & - & 1 \\
\hline RW Cet & 10.1 (B) & A5 & B & 1 & 5.5 & 3.8 & $0.69-0.92$ & - & 1 \\
\hline WY Cet & $9.6(\mathrm{~B})$ & $\mathrm{A} 2$ & BVRI & 16 & 52 & 2.8 & $0.0-1.0$ & $13.2 \mathrm{c} / \mathrm{d}$ & 2 \\
\hline BR Cyg & $9.9(\mathrm{~V})$ & A5 & B & 1 & 4 & 3.9 & $0.79-0.91$ & - & 1 \\
\hline KR Cyg & $9.3(\mathrm{~V})$ & A1 & B & 2 & 10.6 & 3.9 & $0.18-0.36,0.42-0.75$ & - & 2 \\
\hline V456 Cyg & $10.8(\mathrm{~V})$ & A2 & B & 1 & 7.5 & 4.2 & $0.17-0.51$ & - & 1 \\
\hline V466 Cyg & $10.5(\mathrm{~V})$ & A8 & B & 6 & 27.5 & 3.1 & $0.15-0.5,0.55-0.82$ & - & 1 \\
\hline V1034 Cyg & $9.6(\mathrm{~B})$ & $\mathrm{A} 0$ & B & 1 & 3.2 & 3.2 & $0.63-0.76$ & - & 2 \\
\hline RZ Dra & $10.4(\mathrm{~V})$ & A5 & B & 1 & 3.2 & 4.1 & $0.62-0.86$ & - & 1 \\
\hline Z Dra & $10.6(\mathrm{~V})$ & A5 & V & 1 & 3 & 4.1 & $0.68-0.77$ & - & 1 \\
\hline TZ Eri & $9.8(\mathrm{~V})$ & $\mathrm{A} 5 / 6$ & BV & 26 & 70 & 2.7 & $0.0-1.0$ & $18.7 \mathrm{c} / \mathrm{d}$ & 1 \\
\hline TX Her & $8.1(\mathrm{~V})$ & A8 & B & 1 & 3 & 6.5 & $0.79-0.86$ & $?$ & 1 \\
\hline V338 Her & $10.2(\mathrm{~V})$ & A9 & B & 3 & 19.4 & 4.1 & $0.16-0.60,0.63-0.81$ & $?$ & 1 \\
\hline V359 Her & $10.0(\mathrm{~V})$ & F0 & BV & 2 & 10 & 7.1 & $0.16-0.32,0.58-0.65$ & - & 1 \\
\hline IQ Per & $7.73(\mathrm{~V})$ & A7 & BV & 1 & 4.5 & 4.4 & $0.10-0.21$ & - & 1 \\
\hline GH Peg & $9.1(\mathrm{~B})$ & A3 & BVR & 2 & 10.3 & 3.9 & $0.22-0.28,0.63-0.74$ & - & 1 \\
\hline X Tri & $9.0(\mathrm{~V})$ & A3 & BVR & 13 & 25.3 & 2.8 & $0.0-1.0$ & - & 1 \\
\hline RS Tri & $10.3(\mathrm{~V})$ & A5 & B & 2 & 8 & 4.3 & $0.12-0.18,0.66-0.78$ & - & 1 \\
\hline
\end{tabular}

time span, the standard deviation (SD) of the observations (mean value), the phase intervals, the dominant pulsating frequency found and the reference used for quoting the spectral types and the apparent magnitudes of each system. For the majority of the systems, the observations were made in the phase interval where the components were at maximum separation, namely around maxima. Moreover, for the systems which seem to have a pulsating component, we list the most dominant pulsation frequency, while for the ambiguous ones, marked by a (?), the results of frequency analysis are not very convincing, since the possible pulsation frequencies were found to have a $S N R<4.0$, which is less than the pulsation detection limit (Lenz \& Breger 2005) and an amplitude inside the SD limit. The light curves of the largest (data) sets of observations for certain cases of oEA stars are illustrated in figure 1.

\section{The two newly discovered variables}

The data reduction and the frequency analysis methods for studying the new variables are the same as for the eclipsing binaries mentioned above. For GSC 3641-0359 we used the stars GSC 3641-0045 as comparison star (C) and GSC 3641-0037 as check star (K), while for GSC 2673-1583, the GSC 2673-3481 and 

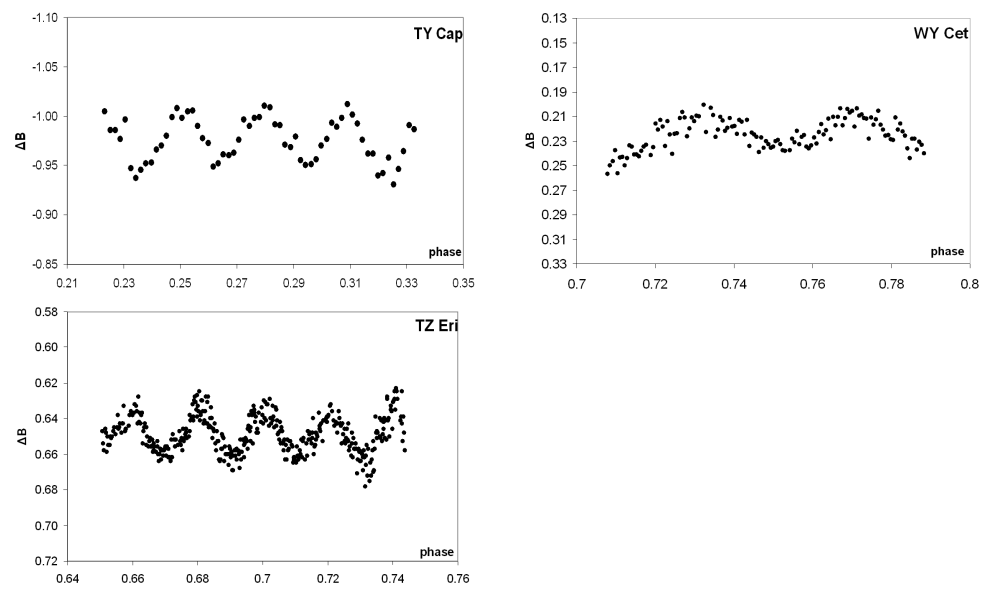

Figure 1: The partial light curves of the systems found to have a pulsating component.

GSC 2673-2979 were used as (C) and (K) stars, respectively. After the first frequency computation, the residuals were prewhitened for the next one. The results of the frequency search for both stars are listed in table 2, listing the identification number of the frequency, the frequency value with its error and the signal-to-noise ratio (SNR) after prewhitening for the previous frequency/ies. The corresponding amplitudes and their standard deviations, derived from a multi-parameter least-squares fit of sinusoidal functions, are also given. The frequency spectra and the Fourier fits to the observational points for the largest (data) sets of observations of each star are presented in figures 3-4.

\section{Discussion and conclusions}

The aim of the present study is to present the results for the pulsational behaviour of some selected eclipsing binaries and for the discovery of two new variable stars. CCD observations of 24 systems, candidate for including pulsating components, have been obtained and analysed using Fourier techniques to search for short-periodic pulsations. The analysis was performed on all available observations. For the brighter targets, we have observations in more than one photometric filter. Nineteen of them do not show any clear evidence of highfrequency pulsations. V338 Her and TX Her are not very convincing cases as the analysis revealed frequencies with amplitudes inside the error limit (SD) and a signal-to-noise ratio less than 4.5. Finally, the systems TY Cap, WY Cet and TZ Eri are clear cases of oEA stars and their dominant frequencies are given in 

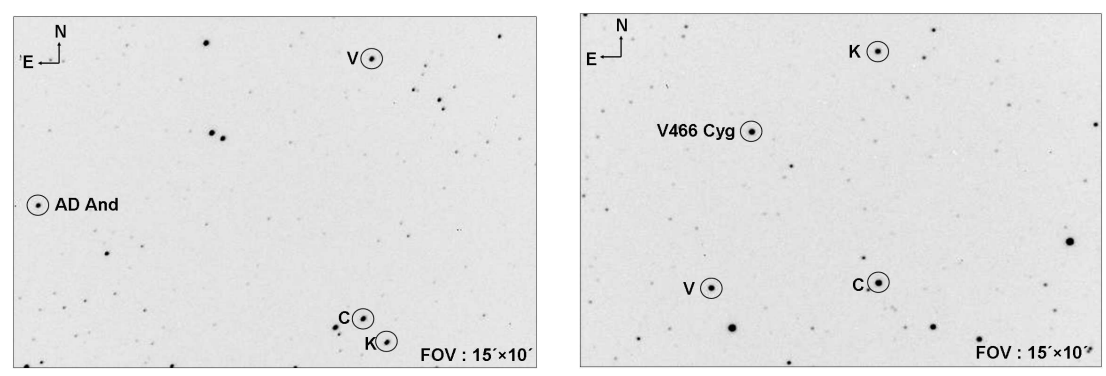

Figure 2: The finding charts of the stars GSC 3641-0359 ( $\mathrm{V}$ in the left part) and GSC 2673-1583 ( $V$ in the right part). The dimensions and the orientation of the field of view and the comparison $(\mathrm{C})$ and check $(\mathrm{K})$ stars are also indicated.

Table 2: The results of frequency analysis for the new variables

\begin{tabular}{|c|c|c|c|c|c|c|c|}
\hline & & \multicolumn{3}{|c|}{ GSC 3641-0359 } & \multicolumn{3}{|c|}{ GSC 2673-1583 } \\
\hline Filter & No & $\begin{array}{l}\text { Freq. } \\
(c / d)\end{array}$ & $\begin{array}{c}\text { Ampl. } \\
(\text { mmag })\end{array}$ & SNR & $\begin{array}{l}\text { Freq. } \\
(c / d)\end{array}$ & $\begin{array}{c}\text { Ampl. } \\
(\mathrm{mmag})\end{array}$ & SNR \\
\hline \multirow{4}{*}{$B$} & $f_{1}$ & $8.3143(4)$ & $15.1(5)$ & 13.56 & $10.0280(4)$ & $9.9(2)$ & 16.20 \\
\hline & $f_{2}$ & $11.2825(7)$ & $8.0(5)$ & 6.32 & $0.4906(5)$ & $7.4(2)$ & 8.34 \\
\hline & $f_{3}$ & $7.6710(10)$ & $5.7(5)$ & 5.36 & $8.8572(12)$ & $3.1(2)$ & 4.94 \\
\hline & $f_{4}$ & $1.2785(17)$ & $3.3(5)$ & 6.30 & & & \\
\hline \multirow{4}{*}{$V$} & $\overline{f_{1}}$ & $8.3126(6)$ & $10.3(5)$ & 11.65 & & & \\
\hline & $f_{2}$ & $11.7081(11)$ & $5.6(5)$ & 5.73 & & & \\
\hline & $f_{3}$ & $7.6700(13)$ & $4.6(5)$ & 5.25 & & & \\
\hline & $f_{4}$ & $1.0119(14)$ & $4.2(5)$ & 6.59 & & & \\
\hline \multirow{4}{*}{$R$} & $f_{1}$ & $8.8859(6)$ & $9.6(5)$ & 13.07 & & & \\
\hline & $f_{2}$ & $11.1346(12)$ & $4.6(5)$ & 5.25 & & & \\
\hline & $f_{3}$ & $7.5222(20)$ & $2.9(5)$ & 4.08 & & & \\
\hline & $f_{4}$ & $0.9887(72)$ & $7.9(5)$ & 10.11 & & & \\
\hline \multirow{4}{*}{ I } & $\overline{f_{1}}$ & $8.8858(5)$ & $9.1(4)$ & 20.26 & & & \\
\hline & $f_{2}$ & $11.0324(9)$ & $5.0(4)$ & 13.65 & & & \\
\hline & $f_{3}$ & 7.9523(13) & $3.7(4)$ & 6.88 & & & \\
\hline & $f_{4}$ & $0.8454(9)$ & $5.2(4)$ & 10.80 & & & \\
\hline
\end{tabular}

table 1. The pulsational behaviour of TZ Eri has been detected by Mkrtichian et al. (2006) and a frequency analysis in detail has been published by Liakos et al. (2009). The detailed analysis of TY Cap and WY Cet will be presented in future work. All the systems were observed near their maxima phases, for at least 3 hours and in some cases, the whole binary's light curve was obtained.

Further observations, using larger telescopes, are certainly needed to clarify the pulsational behaviour of the non-convincing cases.

During the observations of V466 Cyg and AD And, two new variables have been detected and their observations were analysed using the same method. The Fourier analysis revealed that both cases show pulsational behaviour on 

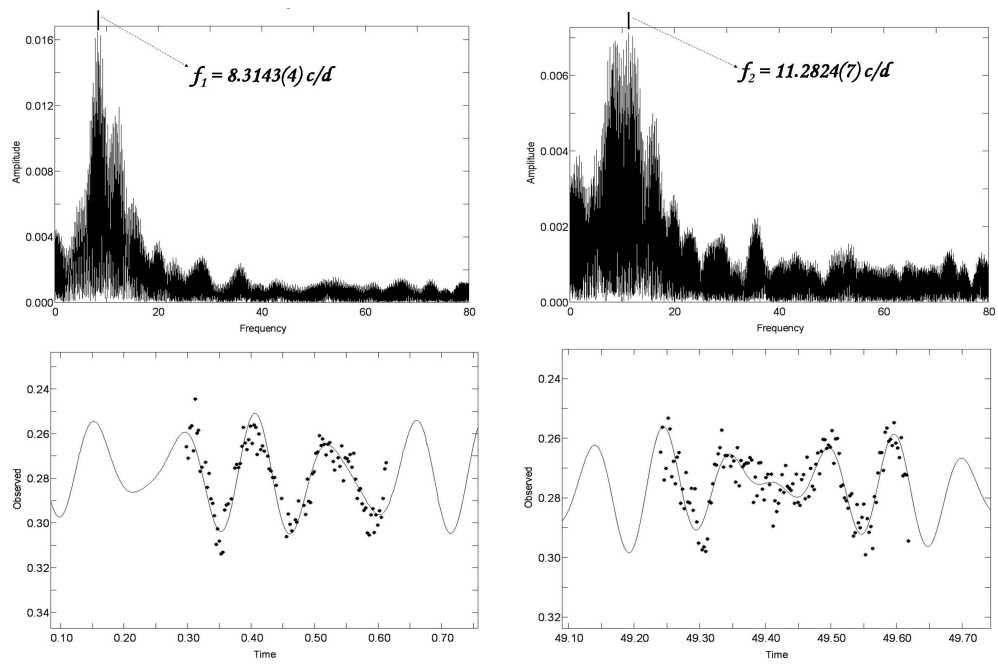

Figure 3: The frequency spectra (upper panels) and the Fourier fits (lower panels) to the observational points for the longest (data) sets of observations of GSC 3641-0359.
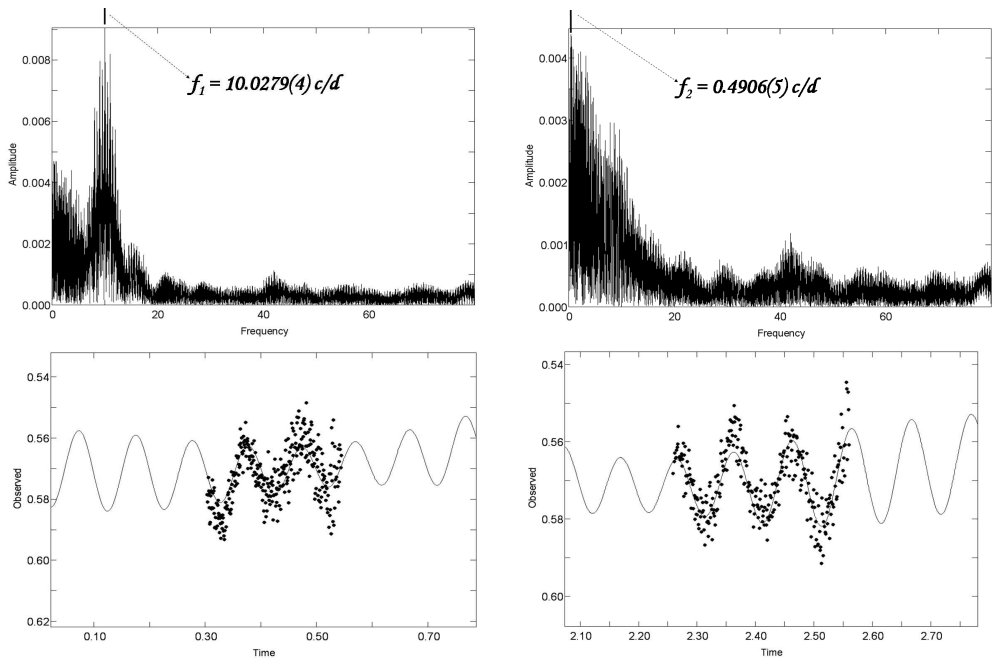

Figure 4: The same as figure 3 for GSC 2673-1583. 
multiperiodic mode. Due to the lack of spectroscopic information, they cannot be categorized. Their pulsational characteristics are similar to those of $\delta$ Scuti stars (5-80 c/d, Breger 2000) and also to those of $\beta$ Cephei stars. We did not find any reasonable frequency values with amplitudes smaller than 2.9 mmag and a signal-to-noise ratio higher than 4 , after prewhitening for the last frequencies in each filter.

GSC 3641-0359 was found to have four pulsation frequencies with the dominant one $8.3143(4) \mathrm{c} / \mathrm{d}$ (with an amplitude of $15.1(5) \mathrm{mmag}$ ), while the period analysis of GSC 2673-1583 revealed a tri-periodic behaviour dominated by the frequency $10.0280(4) \mathrm{c} / \mathrm{d}$ (with an amplitude of 9.9(2) mmag). However, the frequencies $f_{4}$ of GSC 3641-0359 and $f_{2}$ of GSC 2673-1583, since they have a value near $1 \mathrm{c} / \mathrm{d}$, seem to correspond to g-mode pulsations, and could be caused by rotational effects of the star or even by systematic observational errors relevant to atmospheric extinctions (Breger 2005). Of course, the tidal forces of a close companion could also cause these g-mode frequencies, but so far there is no evidence that these stars are members of binary systems. Further spectroscopic observations will certainly reveal the spectral type of each star and will help to detect any close companion(s).

Acknowledgments. We thank the anonymous referee for valuable comments and suggestions that improved the paper. This work has been financially supported by the Special Account for Research Grants No 70/4/5806 of the National \& Kapodistrian University of Athens, Greece.

\section{References}

Breger, M. 2000, ASPC, 210, 3

Breger, M. 2005, ASPC, 333, 138

Hroch, F. 1998, Proceedings of the 29th Conference on Variable Star Research, 30

Kreiner, J. M., Kim, C.-H., \& Nha, I.-S. 2001, An Atlas of O-C Diagrams of Eclipsing Binary Stars / by Jerzy M. Kreiner, Chun-Hwey Kim, Il-Seong Nha. Cracow, Poland: Wydawnictwo Naukowe Akademii Pedagogicznej. 2001.

Mkrtichian, D. E., Kusakin, A. V., Rodriguez, E., et al. 2004, A\&A, 419, 1015

Mkrtichian, D., Kim, S.-L., Kusakin, A. V., et al. 2006, ApSS, 304, 169

Lenz, P., \& Breger, M. 2005, CoAst, 146, 53

Liakos, A., \& Niarchos, P. 2008, IBVS, 5900, in press

Liakos, A., Ulaș, B., Gazeas, K., \& Niarchos, P. 2009, CoAst, 157, 336

Soydugan, E., Soydugan, F., Demircan, O., \& İbanoğlu, C. 2006, MNRAS, 370, 2013 